ISSN 1112-9867

http://www.jfas.info

\title{
INVESTIGATING THE ROLE OF ORGANIZATIONAL HAPPINESS IN TEACHERS' OCCUPATIONAL BURNOUT
}

\author{
A. Hosseinpour Reza ${ }^{1, *}$, B. Esmaeili Leyli ${ }^{2}$ \\ ${ }^{1}$ Assistant Professor of Imam Hossein University, Tehran, Iran \\ ${ }^{2}$ Master of Educational Management
}

Published online: 16 July 2016

\begin{abstract}
The present paper studies the impact of organizational happiness on teachers' occupational burnout. This research employs a descriptive-correlational method. The statistical population is consisted of all 530 elementary school teachers in the city of Kuhdasht. Using Krejcie and Morgan table, sample size is determined to be 226. In order to collect the required data, the Oxford Happiness questionnaire and Maslach Burnout Inventory (MBI) questionnaire have been used. In order to analyze the data and examine the hypotheses, descriptive statistic indices including mean and standard deviation as well as inferential statistics such as Pearson's correlation test have been used. The results indicate that the total scale of organizational happiness and the components of self-concept, life satisfaction, mental preparation, aesthetic emotion, self-efficacy, and hopefulness have a negative and significant relationship with occupational burnout. This implies that as teachers' self-concept, life satisfaction, mental preparation, aesthetic emotion, self-efficacy, and hopefulness improve, their occupational burnout decreases.
\end{abstract}

Keywords: happiness, occupational burnout, teachers

Author Correspondence, e-mail: hosseinpour_reza@yahoo.com

doi: http://dx.doi.org/10.4314/jfas.v8i2s.66 


\section{INTRODUCTION}

\subsection{Concepts and definitions}

In recent years, the experts of human resource productivity have taken an increased interest in the phenomenon of occupational burnout in order to understand its negative impact on the workforce. The occupational burnout occurs in relation with job and damages the mental health. It has three dimensions: 1. Emotional exhaustion is similar to mental pressure variable and refers to the feeling of being under pressure and depletion of emotional resources; 2 . Depersonalization is a negative and cruel response to people who usually receive a service from the individual. This dimension refers to one's negative impression of other people; 3. A reduced sense of personal accomplishment involves negative self-evaluation of one's work Moreover, happiness is an interesting concept which since old ages has attracted the attention of many researchers especially psychologists all over the world (Awa \& Plaumann, 2010).

In terms of its mechanism and effect, happiness is important in two aspects. On one hand, happiness is a positive feeling achieved through a sense of victory and satisfaction. When we have a positive internal feeling, happiness makes the life pleasant. In this sense, the pleasure of happiness neutralizes the inevitable experiences of failure, hopelessness, and other negative emotions. On the other hand, happiness facilitates man's desire to participate in social activities. In this sense, the happiness is a social paste which binds the relationships together (as cited by Drikvandi, 2002). Along with the increasing importance of happiness and its impact on mental health and strength to confront complexities and problems of modern life, the experts and intellectuals as well as the public have changes their opinion about it. The studies reveal that regardless of how it is accomplished, happiness can boost physical health. Happy people feel more secure, make decisions more easily, have better participatory spirit, work harder in their jobs, and feel more satisfied in comparison to other people (Myers, 2002). Pines and Aronson (2009) define job burnout as a state of mental, emotional, and physical fatigue which is caused by long involvement in situations which emotionally demand too much of an individual. According to Goutas (2008), job burnout includes a wide array of symptoms and signs. These signs include weakness, hopelessness, isolation, sensitivity, depression, cynicism, lack of interest, loneliness and separation, emotional exhaustion, and 
depersonalization. Burnout is also closely related to mental and physical diseases. There are powerful evidences which suggest that depression (lack of happiness) and stress may lead to negative consequences such as physical sickness, mental disease, and occupational burnout (Ahmadi, 2013). Happiness refers to living with satisfaction, pleasure, and free from sadness (Sarfejou, 2010). It is one of the six main human emotions (anger, fear, hatred, surprise, sadness, and happiness) (Stewart, Watson, Clark, Obemyer, and Drey, 2010). As a positive concept, happiness is essential for healthy living. Happiness is defined as the victory of positive thoughts over negative, and concentration of emotional weights of life situations (Bikht, 2008). Happiness has positive and consequential effects on different people; these effects increase the power of thinking and improve the quality of communications. In this manner, the feeling of love and devotion is intensified and people tend to help each other. Happy people are more successful in their profession, carry out their tasks better, and have better problem solving abilities (Argyle, 2001).

In recent years, happy climate and organizational happiness in work environment has come to be known as an issue which improves the motivation and efficiency in the employees. It is proven that in environments which are free from tensions between employees and managers and supervisors and conflict between workgroups, the employees have more incentives for faster, better, and more accurate work (Marcus, 2010).

Organizational happiness refers to issues such as intimacy, compassion, honesty, cooperation, and mutual respect between employees. It refers to refraining from managerial intimidation and incentives, prejudice, and absence. Organizational happiness revolves around identification of potential talents and setting grounds for their growth and development. A happy organization tries to identify the causes of lack of work, interest and inaccuracy by the employees. The employees are familiarized with facts of work environment and eliminate the organizational factors which are deemed immoral, inhumane, and culturally problematic (Basadur, 2011).

One of the most important aspects which are influenced by organizational happiness is the qualitative and quantitative level of occupational burnout. Today, job tension and burnout is a new and prevalent problem especially among people working in jobs involving human 
services. The phenomenon of job burnout creates a predisposition for a lot of physical, mental, and emotional diseases which may lead to increased absenteeism, leaving the job, or reduced productivity. Employees suffering from burnout have little motivation and excitement in their work. They become mentally insensitive, depressed, exhausted, and easily offended. They disapprove of everything and person in their work environment and react negatively to suggestions made by others (Myers, 2013).

\subsection{Objectives and hypotheses}

Considering the high importance of organizational happiness and its impact on establishment of constructive mental and spiritual transformations among employees, the present research seeks to study the impact of organizational happiness on teachers' occupational burnout. The main question is: what is the relationship between organizational happiness and teachers' job burnout?

The main hypothesis in this research is: there is a relationship between organizational happiness and teachers' occupational burnout.

The sub hypotheses are:

1. There is a relationship between self-concept and teachers' occupational burnout.

2. There is a relationship between life satisfaction and teachers' occupational burnout.

3. There is a relationship between mental preparation and teachers' occupational burnout.

4. There is a relationship between enthusiasm and teachers' occupational burnout.

5. There is a relationship between aesthetic emotion and teachers' occupational burnout.

6. There is a relationship between self-efficacy and teachers' occupational burnout.

7. There is a relationship between hopefulness and teachers' occupational burnout.

\subsection{Literature and theoretical framework research}

Here, a summary of the research literature and theoretical background is presented:

In order to prevent occupational burnout, first its causes must be identified. Also, the factors related to this issue should be identified so that in future they can be confronted (Salahian et al. 2011). Despite many attempts, the factors affecting job burnout have not yet been accurately identified (Salahian et al. 2013; Khakpour \& Birashk, 1998). Variables such as job stress, role transparency, and task heaviness are usually considered to be the predictors of occupational 
burnout.

Job stress: The first instance of discussion about job stress pertains to Hans Sellier who is known as the father of psychological stress. Job stress is a state in which job-related factors interfere with an individual's mental and psychological health. the statistical findings reveal that stress and its side effects ruin hundreds of work-days every year. On an average, a million employees avoid going to work every day due to side effects of stress. Also, $4 \%$ of work-hours are wasted because of absenteeism due to job stress and job dissatisfaction.

Role transparency: It is yet another factor which is related to job burnout. Role transparency and ambiguity are used interchangeably in the research literature; they refer to an individual's perception of his/her role.

Role over-load: it is an important factor which creates emotional pressure in the workplace. In today's competitive environment, organizations seek to downsize their business; this strategy executed to have less number of employees for doing more work in organizations and firms (Salahian et al. 2011).

Maslach (2001) considered the causes of burnout to be the high pressure and volume of work, lack of competence and awareness about the job process, lack of reward, weakness of communication between employees, unfair wage payment, lack of individual value, and double standards between personal and occupational values (Toubaei et al. 2009). As cited by Kraft (2006), Freudenberger identifies 12 factors resulting in staff burnout including obligation to prove oneself, too much work, ignoring the needs, movement of conflicts, reconsideration of values, denial of emerging problems, isolation and keeping to oneself, observable behavioral changes, depersonalization, feeling of emptiness and general lack of interest, depression, and psychological and physical deterioration.

Some researchers identify the cause of job burnout to be long involvement and exposure to stress and mental pressure; they classify the factors leading to mental pressure and depression into three categories:

1) Environmental factors including economical uncertainty, political uncertainty, social transformations, and mistrust in technology. 
2) Personal factors including the extent to which changes and incidents occur in one's life, personality, perception, personal differences, and values.

3) Organizational factors which are comprised of:

a.Factors related to the organization itself such as organizational policies and structure, lack of job security and/or possibility for progress and promotion, organizational processes, excessive and offensive control, and conflict in goals and objectives.

b. Factors related to the job such as role over-load, conflict and discrepancy in responsibilities, lack of social support, geographical mobility, and role under-load (Arab et al. 2012).

A meta-analysis based on five personality models between 1993 and 1997 shows that neuroticism, extraversion, and conscientiousness have the closest relationship with occupational burnout (Poursadegh, 2012). Job burnout depends on various factors such as poor choice, lack of adequate skills, inappropriate relationships with coworkers, and personal and social tension-creating factors. In general, job burnout is a serious mental-physical problem which originates from neurological tension and pressure. Therefor, the factors creating tension and conflict i.e. personal, organizational, and socio-economic factors have an effective role in emergence of occupational burnout.

In the study of environmental factors which create burnout, it is important to note the general characteristics of the institutions which intensify occupational burnout. Such characteristics often include role over-load and vastness, role ambiguity, extensive work hours and work volume, and unpleasant and ambiguous policies; most of such institutions are governmental organizations (Masoudi et al. 2008). Dorman (2003) claims that a lot of studies have focused on job burnout and numerous factors have been identified in that regard; however, the primary cause of occupational burn out involves the employee being under mental and neurological pressure for too long.

Since happiness is a basic human emotion, everyone experiences it to some extent. However, the definition of happiness is not as simple as its experience. Leobomirky, Sheldon \& Schekade (2005) define happiness as excitement, joy, and delight along with the feeling of 
having a good, significant, and valuable life. According to this definition, happiness is a subjective and intrinsic phenomenon. Bradburn (2001) believes that happiness is the extent of positive emotion minus the negative feelings. The negative emotion in Bradburn's definition is measured by Eysenck mental illness scale and Beck's depression test. The most comprehensive and practical definition of happiness is yet proposed by Winhoven (1984): happiness is the extent to which an individual likes his/her life as being delightful and pleasant (Winhoven, 1984; quoted by Abedi 2005). A lot of studies have been carried out regarding the relationship between demographical and environmental factors and happiness. These studies have been started by Katryl (1965), Bradburn (1969), Komeili, Karnvrs \& Rabvz (1976), and Winhorn et al. (1994) (Eddington \& Shuman, 2004).

The sources of happiness are different for different sexes. Men are usually impressed by themselves, their job, and economic prosperity, while women are affected by the children and family health. Women demonstrate more signs of depression and negative emotions in comparison to men. However men and women both report the same level of general satisfaction. The reason might be that women are better capable of accepting negative feelings while men basically deny having such negative emotions (Eddington \& Schumann, 2004).

The most important factor which determines happiness and job satisfaction is autonomy, or freedom and independence toward the direct control which exists in an individual's job. Job satisfaction is one of the most effective factors contributing to well-being and happiness. The concept of flow was first introduced by Mihalyi (1997) into the literature of happiness and well-being. Flow refers to the extremely joyful moments in which what an individual experiences is in alignment with what he desires and thinks. He believes that people experience more happiness during their work and it seems that job satisfaction is one of the most significant predictors of happiness and well-being (Eysenck, 1996; translated by Firouzbakhti \& Beigi, 1999).

Characteristics of happy and healthy people

1- Happy people usually tend to evaluate themselves better and remember positive life events more that the negative one. They are also better in life decision-making (Carr, 2004; translated by Pashasharifi et al. 2006). 
2- Happy people have an optimistic and hopeful approach in their processing of information, that is, they interpret the information in a way that leads to their happiness and delight (Schwarz and Strack, 1991).

3- Happy people have higher self-esteem and self-respect. They love themselves. Such individuals are highly concerned about morality and behave rationally (Janov-Bullman, 1989; Myers, 1993; as quoted by Nouri, 2006).

4- Happy people are optimistic. These individual are more successful and healthier than pessimistic people (Dember and Brook, 1989; Sligman, 1991; as quoted by Karami Nouri et al. 2002).

5- Happy people are extravert. They are well-capable in communication and cooperation with others; they enjoy their lives as well as lives of other people and take delight in different personal or social jobs (Diner et al. 1992; as quoted by Karami Nouri et al. 2002).

6- Happy people experience less emotional frustration, deterioration, and absenteeism; they are less probable to leave their jobs (Baum \& Leobomirsky, 2007; as quoted by Zarei Matin et al. 2009).

7- Happy people tolerate the daily life problems better (Lama and Cutler, 2004; as quoted by Sheidaei, 2011).

8- Happy people are more responsible and accept criticism in a better manner (Sobhaninia, 2005; as quoted by Sheidaei, 2011).

9- Happy people are more creative (Safari Shali, 2008; as quoted by Sheidaei, 2011).

There is a moderate level in between strong and weak levels of happiness. People with moderate level of happiness are well-experienced yet have little understanding of the organization. In his studies, Alpert point out the little extent of employees' involvement with organizational affairs. The quality of different levels of happiness and its results and consequences on employees and organization are represented in the table below (Saroughi, 1996).

In fact, low levels of happiness are not desirable for the individual, neither are they fruitful for the organization. In a situation like this, the benefits of an employee's work in the organization are lost and the organization is forced to withstand incompetent and disloyal 
employees. In higher levels of happiness, it can be said that positive consequences outweigh negative results. Individuals may progress rapidly in their jobs and increase the production level; however they are forced to tolerate limitations in their personal, family, and social life. In a situation like that, the organization's conditions may not be satisfactory for the individuals. Therefor the organization might lose its flexibility and impose its weight on hardliners and extremist individuals; in this case, the organization is prone to illegal and immoral actions. All in all, it seems that there is no linear relationship between happiness and desired outcomes. This relation is more like an arch and the best kind of relationship exists in the middle of the arch where the employees' needs and organization's expectations are at the moderate level (Samad, 2005).

\subsection{History Research}

In the study of the relationship between organizational happiness, job satisfaction, and job performance, Samad (2005) investigated 584 employees in Telekom Malaysia, and reported that job satisfaction did play a positive moderating role in the relationship between organizational commitment and employee performance. Thus, organizational commitment and job satisfaction are correlated and important attitudes in assessing employees' intention to quit and the overall contribution of the employee to the organization.

In a study titled "the relationship between job satisfaction and organizational happiness", Proof (2006) studies 132 university employees in Turkey. The results of correlation analysis indicate that concerning the job and the organization, there is a positive correlation between job satisfaction and emotional and normative happiness.

A study by Chen (2004) titled "Examining the effect of organization culture and leadership behaviors on organizational happiness, job satisfaction, and job performance" investigates 451 employees of service and industry organizations in Taiwan. The results are as follows: 1Organizational happiness facilitates the relationship between leadership behavior and job performance in that culture; 2- effective and idealistic leadership and new culture have positive relationships with organizational happiness.

A study by Bodamusa (2005) titled "the impact of performance evaluation on organizational happiness" investigates 400 employees in Botswana. In this research, employee evaluation or 
lack there of, and its impact on organizational happiness is investigated. The results indicate that normative happiness is positively and considerably related to lack of performance evaluation. Both normative and continuance commitment have a positive and significant relationship with performance evaluation. The study proves that performance evaluation affects organizational commitment and happiness.

Boles et al. (2007) study the relationship of facets of salesperson job satisfaction with affective organizational happiness. Findings demonstrate that different types of satisfaction are related to organizational happiness. Further, various facets of satisfaction such as pay and promotion are more important to men than to women. Women find that satisfaction with co-workers is more closely related to organizational commitment than it is for men.

Coopers and Laybrand study large firms in the years 2013 and 2014. They conclude that there is a direct relationship between job burnout, organizational climate, and workplace happiness. As the level of happiness and well-being rises to desired levels, the employees' job satisfaction doubles.

The studies by Simons (2013) reveal that job characteristics highly affect the levels of occupational burnout. According to him, the identification of psychological factors in workplace is the key to a correct perception and understanding of job satisfaction.

The study by Barton \& Folkard (2012) reveals the impact of job characteristics such as mental satisfaction and happiness on reduction of occupational burnout. The job characteristics which affect job satisfaction are classified into four categories i.e. work pace, work repetition, work schedule, and task-related characteristics.

Mets and Cherry (2007) classify other factors related to job satisfaction. They suggest that the following factors can increase or decrease job burnout. 1- Interpersonal relationships: the quality of relationships between employees can affect job satisfaction; 2- organizational structure and climate: the structures which offer more decision-making power to their employees create less stress; 3- human resource management method: different methods of human resource management have different impacts on the stress imposed on employees; 4technology and tangible characteristics: as far as these factors are concerned, the stress is heightened when the minimum life requirements are not provided. 


\section{1-5 Methodology}

In terms of purpose, this is an applied study, and in terms of method it is a correlational-type research. The statistical population is consisted of all 530 teachers (320 men and 210 women) working in Kuhdasht County in 2014-15 school year. Using Krejcie-Morgan table, the sample size is determined to be 226; the sample members have been selected using relative stratified random sampling method (132 men and 94 women). The required data is collected through field study as well as library study. One of the instruments of data collection is the Oxford Happiness questionnaire which has been designed by Argyle et al. (1989) in order to assess happiness and well-being. In a study titled "the reliability, credibility, and normalization of Oxford Happiness questionnaire", Zarei (2009) tested this questionnaire on 1021 high-school students in Zanjan. Using test-retest method, the reliability of this questionnaire was determined to be 0.78 . The Cronbach's Alpha value for the first test was 0.83 and the Alpha value for the retest was 0.86 . The values of correlation coefficient of this questionnaire with the five personality factors of neuroticism, extraversion, openness-to-experience, agreement, and conscientiousness are $-0.30,0.43,0.25,0.14$, and 0.37 respectively; all the obtained values have been statistically significant. The other instrument of data collection in this research is Maslach \& Jackson burnout inventory questionnaire (MBI). Using Cronbach's Alpha, Maslach and Jackson determined the internal consistency of the MBI questionnaire to be 0.83 for frequency, and 0.84 for intensity. In the present study, the reliability of the MBI questionnaire has been assessed using Cronbach's Alpha coefficient. The Alpha values for the whole questionnaire and the subscales of emotional exhaustion, personal performance, depersonalization, and conflict are $0.89,0.86,0.87$, and 0.88 respectively.

In order to analyze the data descriptive statistics including minimum, maximum, mean, standard deviation, frequency distribution tables, and related charts have been used; also inferential statistics including Kolmogorov-Smirnov test to determine normality of distribution as well as Pearson's correlation test to determine the relationship between two variables have been employed.

\section{6 research concept model}

Figure 1 demonstrates the concept model extracted based on the discussed theoretical basis 
and research literature.

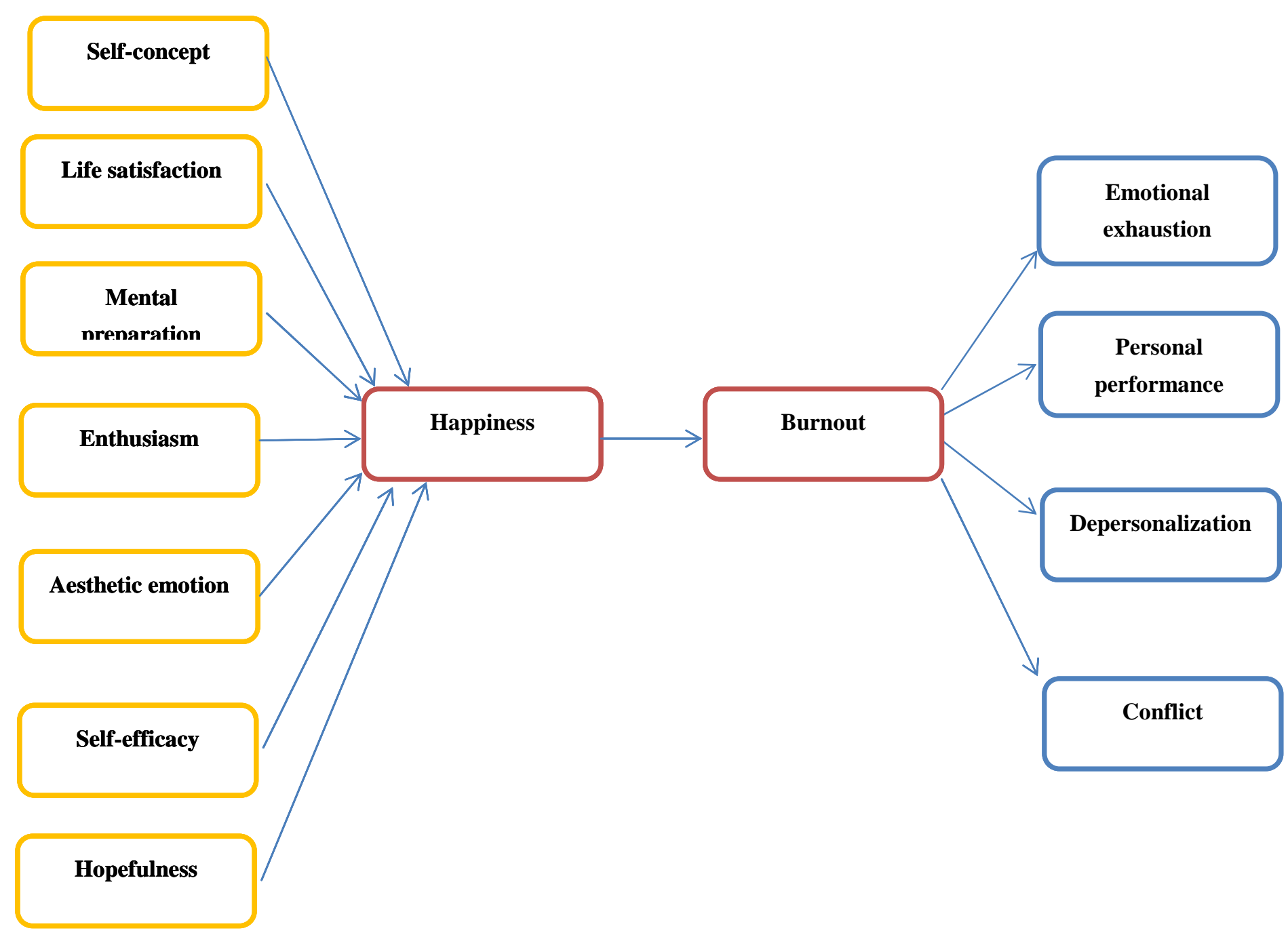

Fig.1. Research concept model

\section{RESULTS AND DISCUSSION}

1- Description of scores of organizational happiness and occupational burnout

The table below represents mean and standard deviation of the participants' scores on different variables 
Table 1: mean, standard deviation, number, minimum and maximum, and range of variations

\begin{tabular}{crrrrrr}
\multicolumn{7}{c}{ for each variable } \\
\hline Variable & Mean & \multicolumn{1}{c}{$\begin{array}{c}\text { Std. } \\
\text { deviation }\end{array}$} & Number & Max & Min & $\begin{array}{r}\text { Range of } \\
\text { variations }\end{array}$ \\
\hline Happiness & 62.27 & 13.03 & 226 & 84 & 22 & 62 \\
Self-concept & 18.92 & 4.04 & 226 & 24 & 2 & 22 \\
Life satisfaction & 70.30 & 1.64 & 226 & 9 & 2 & 7 \\
Mental preparation & 8.97 & 1.92 & 226 & 12 & 1 & 11 \\
Enthusiasm & 4.22 & 1.19 & 226 & 6 & 0 & 6 \\
Aesthetic emotion & 11.59 & 2.77 & 226 & 15 & 2 & 13 \\
Self-efficacy & 8.91 & 2.41 & 226 & 12 & 0 & 12 \\
Hopefulness & 4.78 & 1.43 & 226 & 6 & 0 & 6 \\
Occupational burnout & 73.86 & 12.60 & 226 & 111 & 48 & 63 \\
\hline
\end{tabular}

As demonstrated in the table above, the values of mean and standard deviation for organizational happiness are 67.27 and 13.03 respectively. Also in the subscales, these values are 18.92 and 4.04 for self-concept, 7.30 and 1.64 for life satisfaction, 8.97 and 1.92 for enthusiasm, mental preparation, 4.22 and 1.19 for mental preparation, 11.59 and 2.77 for aesthetic emotion, 8.91 and 2.41 for self-efficacy, and 4.78 and 1.43 for hopefulness. Also, the values of mean and standard deviation for the whole scale of occupational burnout are 73.86 and 12.60 respectively.

2- Inferential findings

In this research, in order to determine the normality of the variables, the single-sample Kolmogorov-Smirnov test has been used. The results of the examination of normality hypothesis are presented in table 2. 
Table 2: Test of normality of research variables

\begin{tabular}{|c|c|c|}
\hline Variables & Kolmogorov-Smirnov & Sig. \\
\hline Happiness & 1.29 & 0.11 \\
\hline Self-concept & 1.23 & 0.13 \\
\hline Life satisfaction & 1.18 & 0.16 \\
\hline Mental preparation & 1.17 & 0.17 \\
\hline Enthusiasm & 1.30 & .10 \\
\hline Aesthetic emotion & 1.31 & 0.09 \\
\hline Self-efficacy & 1.15 & 0.16 \\
\hline Hopefulness & 1.24 & 0.13 \\
\hline Occupational burnout & 1.10 & 0.20 \\
\hline
\end{tabular}

As indicated in the table above, the level of significance of the research variables is above the alpha level $(\alpha=0.05)$. Therefor data distribution is normal and Pearson's correlation test can be used to examine the relationship between variables.

The main hypothesis: there is a relationship between organizational happiness and occupational burnout among elementary school teachers of Kuhdasht.

In order to examine the relationship between organizational happiness and occupational burnout among elementary school teachers of Kuhdasht, Pearson's correlation test has been employed and the results are demonstrated in table 3. 
Table 3: The results of the correlation between happiness and its subscales and occupational burnout

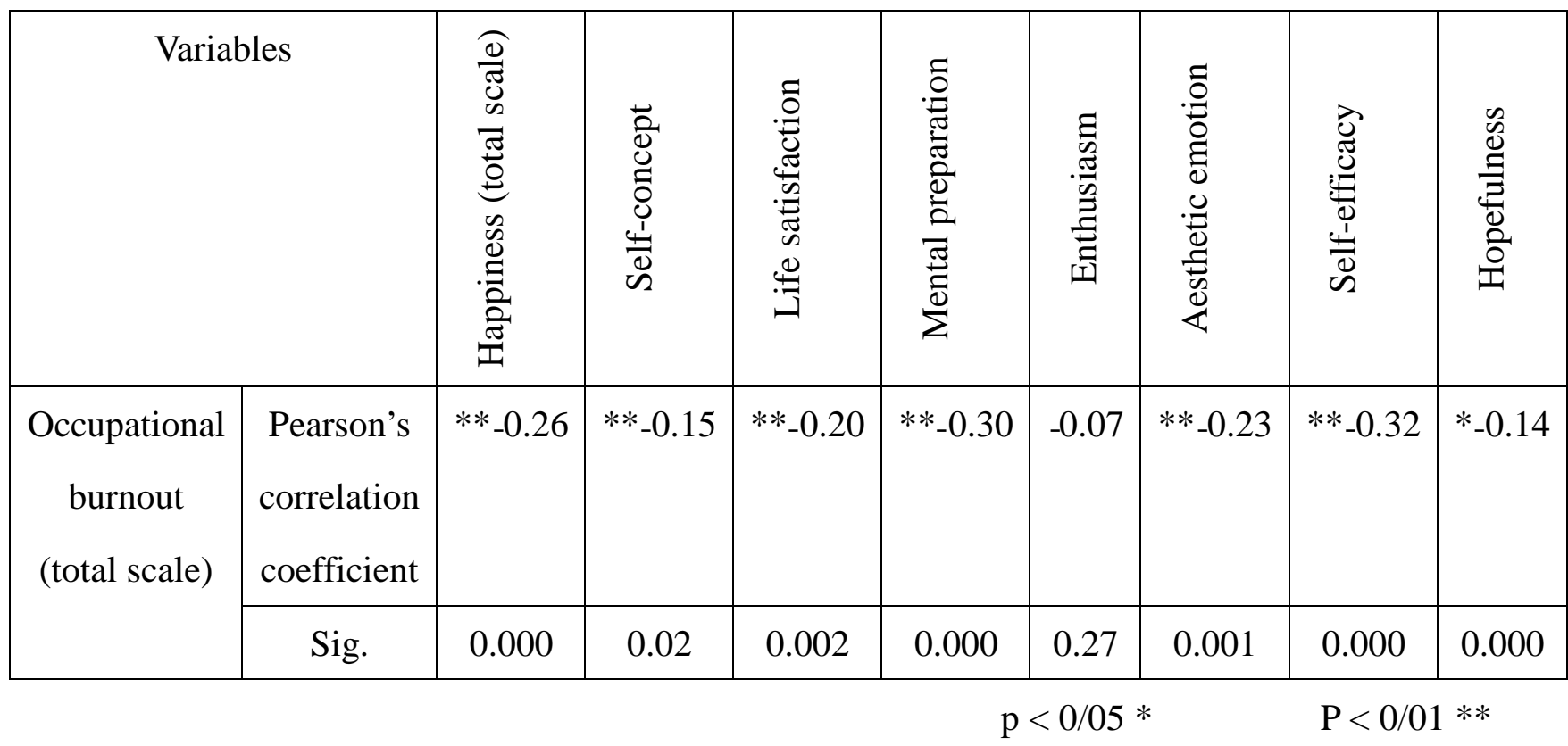

Considering the results represented in the table above, the total scale of occupational burnout has a negative and significant relationship with happiness and its subscales i.e. self-concept, life satisfaction, mental preparation, aesthetic emotion, self-efficacy, and hopefulness.

Examination of the sub-hypotheses:

1- There is a relationship between self-concept and occupational burnout among elementary school teachers of Kuhdasht.

Table 4: The results of the correlation between self-concept and total scale of occupational burnout

\begin{tabular}{|c|c|c|}
\hline \multicolumn{2}{|c|}{ Variables } & Self-concept \\
\hline $\begin{array}{c}\text { Occupational } \\
\text { burnout }\end{array}$ & Pearson's & $* *_{-} 0.15$ \\
& $\begin{array}{l}\text { correlation } \\
\text { coefficient }\end{array}$ & \\
\cline { 2 - 3 } & Sig. & 0.02 \\
\hline
\end{tabular}

Considering the results represented in the table above, there is a negative and significant relationship between self-concept and occupational burnout $(\mathrm{p}<0.05)$. 
2- There is a relationship between life satisfaction and occupational burnout among elementary school teachers of Kuhdasht.

Table 5: The results of the correlation between life satisfaction and total scale of occupational burnout

\begin{tabular}{|c|c|c|}
\hline \multicolumn{2}{|c|}{ Variables } & Life satisfaction \\
\hline $\begin{array}{c}\text { Occupational } \\
\text { burnout }\end{array}$ & $\begin{array}{c}\text { Pearson's } \\
\text { correlation } \\
\text { coefficient }\end{array}$ & $* *_{-} 0.20$ \\
\cline { 2 - 3 } & Sig. & 0.002 \\
\hline
\end{tabular}

Considering the results represented in the table above, there is a negative and significant relationship between life satisfaction and occupational burnout $(\mathrm{p}<0.01)$.

3- There is a relationship between mental preparation and occupational burnout among elementary school teachers of Kuhdasht.

Table 6: The results of the correlation between mental preparation and total scale of occupational burnout

\begin{tabular}{|c|c|c|}
\hline \multicolumn{2}{|c|}{ Variables } & Mental preparation \\
\hline $\begin{array}{c}\text { Occupational } \\
\text { burnout }\end{array}$ & Pearson's & $* *_{-} 0.30$ \\
& $\begin{array}{c}\text { correlation } \\
\text { coefficient }\end{array}$ & \\
\cline { 2 - 3 } & Sig. & 0.000 \\
\hline
\end{tabular}

Considering the results represented in the table above, there is a negative and significant relationship between mental preparation and occupational burnout $(\mathrm{p}<0.05)$.

4- There is a relationship between enthusiasm and occupational burnout among elementary school teachers of Kuhdasht. 
Table 7: The results of the correlation between enthusiasm and total scale of occupational burnout

\begin{tabular}{|c|c|c|}
\hline \multicolumn{2}{|c|}{ Variables } & Enthusiasm \\
\hline $\begin{array}{c}\text { Occupational } \\
\text { burnout }\end{array}$ & Pearson's & -0.07 \\
& $\begin{array}{c}\text { correlation } \\
\text { coefficient }\end{array}$ & \\
\cline { 2 - 3 } & Sig. & 0.27 \\
\hline
\end{tabular}

Considering the results represented in the table above, there is no significant relationship between enthusiasm and occupational burnout $(\mathrm{p}>0.05)$.

5- There is a relationship between aesthetic emotion and occupational burnout among elementary school teachers of Kuhdasht.

Table 8: The results of the correlation between aesthetic emotion and total scale of occupational burnout

\begin{tabular}{|c|c|c|}
\hline \multicolumn{2}{|c|}{ Variables } & Aesthetic emotion \\
\hline $\begin{array}{c}\text { Occupational } \\
\text { burnout }\end{array}$ & $\begin{array}{c}\text { Pearson's } \\
\text { correlation } \\
\text { coefficient }\end{array}$ & $* *_{-} 0 / 23$ \\
\cline { 2 - 3 } & Sig. & $0 / 001$ \\
\hline
\end{tabular}

Considering the results represented in the table above, there is a negative and significant relationship between aesthetic emotion and occupational burnout $(\mathrm{p}<0.01)$.

6- There is a relationship between self-efficacy and occupational burnout among elementary school teachers of Kuhdasht.

Table 9: The results of the correlation between self-efficacy and total scale of occupational burnout

\begin{tabular}{|c|c|c|}
\hline \multicolumn{2}{|c|}{ Variables } & Self-efficacy \\
\hline \multirow{2}{*}{$\begin{array}{c}\text { Occupational } \\
\text { burnout }\end{array}$} & Pearson's & $* *-0 / 32$ \\
& $\begin{array}{c}\text { correlation } \\
\text { coefficient }\end{array}$ & \\
\cline { 2 - 3 } & Sig. & $0 / 000$ \\
\hline
\end{tabular}


Considering the results represented in the table above, there is a negative and significant relationship between self-efficacy and occupational burnout $(\mathrm{p}<0.01)$.

7- There is a relationship between hopefulness and occupational burnout among elementary school teachers of Kuhdasht.

Table 9: The results of the correlation between hopefulness and total scale of occupational burnout

\begin{tabular}{|c|c|c|}
\hline \multicolumn{2}{|c|}{ Variables } & Hopefulness \\
\hline \multirow{2}{*}{$\begin{array}{c}\text { Occupational } \\
\text { burnout }\end{array}$} & Pearson's & $*_{-} 0 / 14$ \\
& $\begin{array}{c}\text { correlation } \\
\text { coefficient }\end{array}$ & \\
\cline { 2 - 3 } & Sig. & $0 / 033$ \\
\hline
\end{tabular}

Considering the results represented in the table above, there is a negative and significant relationship between hopefulness and occupational burnout $(\mathrm{p}<0.05)$.

\section{CONCLUSION AND DISCUSION}

There is a relationship between organizational happiness and occupational burnout among elementary school teachers of Kuhdasht.

The results indicate that occupational burnout has a negative and significant relationship with happiness and its subscales i.e. self-concept, life satisfaction, mental preparation, aesthetic emotion, self-efficacy, and hopefulness. Unfortunately, the existing research literature focuses more on the abnormal and negative impacts of behavior on mental health and deals little with the role of happiness in mental health. Yet, happiness can be a good predictor of people's prosperity. In the end, happiness has a negative and significant relationship with job burnout because happy people feel less frustrated in their workplace. Of course it should be mentioned that lack of happiness does not necessarily lead to serious mental problems; but it works as a trigger which may lead the individual toward anxiety which is the most common cause of depression and communication disorder (Pardakhtchi, Ahmadi, and Arezoumandi, 2012).

There is a relationship between self-concept and occupational burnout among elementary school teachers of Kuhdasht. Considering the results, there is a negative and significant 
relationship between self-concept and occupational burnout. This finding corresponds to that of the study by Raoufi (2013).

There is a relationship between life satisfaction and occupational burnout among elementary school teachers of Kuhdasht. This finding which suggests there is a negative and significant relationship between self-concept and occupational burnout is to some extent in alignment with the conclusion made by Asghari et al. (2012).

There is a relationship between mental preparation and occupational burnout among elementary school teachers of Kuhdasht. Human's mental and psychological aspect plays an important part in his growth and progress. This finding which suggests there is a negative and significant relationship between mental preparation and occupational burnout is to some extent in alignment with the conclusion made by Asghari et al. (2012).

There is a relationship between enthusiasm and occupational burnout among elementary school teachers of Kuhdasht. Considering the results, there is no significant relationship between enthusiasm and occupational burnout. No other study was found to suggest a similar conclusion.

There is a relationship between aesthetic emotion and occupational burnout among elementary school teachers of Kuhdasht. Considering the results, there is a negative and significant relationship between aesthetic emotion and occupational burnout. No similar research conclusion was found to either support or contradict this conclusion.

There is a relationship between self-efficacy and occupational burnout among elementary school teachers of Kuhdasht. Considering the results, there is a negative and significant relationship between self-efficacy and occupational burnout. This conclusion is in alignment with the findings of the studies by Scalewick and Scalewick (2007), Agid and Short (2006), and Asghari (2006). As mentioned, the feeling of self-efficacy leads to competence and success. Effective performance needs skills as well as the belief in one's skills. Therefor, self-efficacy helps individuals to use their skills in confrontation with their problems in an efficient way. It increases the perception of personal success in people. In other words, self-efficacy does not just refer to having a set of skills; it also refers to a person's belief in his/her skills to carry out the tasks in different situations. 
There is a relationship between hopefulness and occupational burnout among elementary school teachers of Kuhdasht. Considering the results, there is a negative and significant relationship between hopefulness and occupational burnout. This conclusion is in alignment with the findings of the study by Ekrami, Rezaei, and Bayani (2014) who conclude that there is a negative and significant relationship between hopefulness at work and educational burnout among university students. The relationship between hopefulness and optimism and occupational burnout has been discussed to a great extent in the literature of psychology. It seems that hopefulness decreases teachers' burnout and optimism at work leads to better performance in educational activities. It must be noted that the present research only studies the relationship between happiness and occupational burnout, while there are other important variables such as age, social support, control source, life style, socio-economic status, and etc. which are related to occupational burnout. Moreover, this study has been conducted on teachers in the city of Kuhdasht; since occupational burnout and other job-related issues are also affected by organizational culture and climate, leadership styles, and similar factors, and considering the fact that these factors may vary from one organization to another, therefor one must be careful in generalization and extending the findings of this research to other organizations.

\section{REFERENCES}

Aghajani M, Tizdast T, Abas Ghorbani M, Bajvar M, , the relationship between psychological hardiness and nurses' burnout, comprehensive nursing, 2013,23(7).

Asadi M, Shafiabadi A, Panahali A, Habibolahzadeh H, The Effectiveness of Group Logo Therapy in Reducing Job Burnout, Thought and Behavior in Clinical Psychology, 2011,6(22). Awa, W. L, Plaumann, M., Walter, U, Burnout prevention: A review of intervention programs. Patient Education and Counseling, 2010, 78: 184-190.

Beigifard S, 1999, the relationship between hardiness and social support and job burnout in employees of Shiraz well-being rehabilitation centers, M.A. thesis, University of Social Welfare and Rehabilitation Sciences

Dorman, J. Testing A Model for Teacher Burnout, Australian Journal of Educational \& 
Developmental Psychology, 2003, 3: 35-47.

Egyed, Carla \& Short, Rick. Jay. Teacher self - Efficacy, Burnout, Experience and Decision to Refer a Disruptive student. School psychology international, 2006,27(4): 462 - 474

Ekrami A, Rezaei, Tehri, Bayani A, relationship between hopefulness at work and educational burnout among university students, Knowledge and health periodical, 2014, 10(1) :20-32.

Etemadi O, Faghih M, investigating the causes of job burnout in elementary school teachers of Isfahan, Occupational and organizational consulting, 2008, 2(2).

Goutas, Lydia, Burnout. The Journal of European Medical Writes Association, 2008, 3: 135-138.

Goutas, Lydia. Burnout. The Journal of European Medical Writes Association, 2008, 3: $135-138$.

Grau-Alberola E. Gil-monte PR. Garcia-juesas JA. Figuriredo-Ferraz H. Incidence of burnout in Spanish nursing professionals: A Longitudinal study. International Journal of Nursing Studies; 2010, 47(8): 1013-1020.

Kraft, U. (2006). "Burned out: Your job is extremely fulfilling. It is also extremely demanding. And you feel overwhelmed. You are not alone”, Scientific American Mind, June/July 2006: 29-33.

Kumar S, Fischer J, Robinson E, Hatcher S, Bhagat RN. Burn out and job satisfaction. In newzeland psychiatrics. A national study. Int J Soc psychiatr; 2007, 53:306-16.

Lin C. Culture shock, social support, and intercultural competence: an investigation of a Chinese Student Organization on a U.S. campus. J Intercult Commun Res; 2006, 35:117-37. Maslach C, Schaufeli WB, Leiter MP. Job burnout. Annu Rev Psychol; 2001, 52: 397-422. Masoudi Sh, Etemadifar R, Afzali M, Kheiri F, Hassanpour Dehkordi A, the factor affecting job burnout in nurses working at selected private hospitals in Tehran, Nursing studies, 2008, $3(8)$.

Mazloumi S, Saeedi M, Vahedian M, Jalalpour Z, Kiani M, the impact of occupational burnout on social support and self-esteem in healthcare workers in Yazd city, Teb-Kar expert scientific periodical, 2013, 5(1).

Miler K, ed. (2006). Organizational communication: approaches and process. 4th ed. Belmont, 
CA: Wadsworth.

Myers, D.G, (2002). Happy and Healthy. Http: // abclocal.Go.Com / 68 - wls / News / 012802

- hs - happy healthy.Htm 1

Pines, A, Aronson, E, Kafry, D. (2002), Burnout: From Tedium to Personal Growth. Free Press, New York.

Poursadegh $\mathrm{N}$, the strategies to confront mental pressure and personality characteristics in relation with employees' job burnout, strategic management studies, 2012, 10

Raoufi R, 2013, the relationship between teaching style and self-concept and job burnout among English teachers, M.A. thesis, Ferdowsi University

Rashedi V, Foroughan M, Hosseini M, the relationship between organizational culture and job burnout in employees of Tehran welfare centers, health improvement management, 2012, No.1, No.2

Scott, C.R. (2001). "Communication, social support and burnout: A brief literature review", Copyright 2002 by Susan, K.S.

Skaalvik, Einar. M \& skaalvik, Sidsel.Dimensions of Teacher Self -Efficacy and Relations with strain Factors, Perceived Collective Teacher Efficacy, and Teacher Burnout. Journal of Educational Psychology, 2007, 99(3): 611-625.

Toubaei Sh, Daghigh Afkat M, Haghshenas H, the relationship between job burnout, mental health, and personality traits among dentists, Shiraz University Dentistry magazine, $2009,10,(4)$

Zopiatis, A. Constanti, P. "Leadership styles and burnout: is there an association?" International Journal of Contemporary Hospitality Management, 2010, 22, (3): 300-320.

\section{How to cite this article:}

Hosseinpour Reza A, Esmaeili Leyli B. Investigating the role of organizational happiness inteachers' occupational burnout. J. Fundam. Appl. Sci., 2016, 8(2s), 1444-1465. 